Abstracta Iranica Abstracta Iranica

Revue bibliographique pour le domaine irano-aryen

Volume 42-43 | 2021

Comptes rendus des publications de 2019-2020

Ashk P. Dahlén. Achaemenid Anatolia: Persian

Presence and Impact in the Western Satrapies 546-330

$B C$. Procedings of an International Symposium at the Swedish Research Institute in Istanbul, 7-8 September 2017

Rémy Boucharlat

\title{
OpenEdition
}

Édition électronique

URL : https://journals.openedition.org/abstractairanica/53551

DOI : 10.4000/abstractairanica.53551

ISSN : $1961-960 X$

Éditeur :

CNRS (UMR 7528 Mondes iraniens et indiens), Éditions de l'IFRI

Référence électronique

Rémy Boucharlat, « Ashk P. Dahlén. Achaemenid Anatolia: Persian Presence and Impact in the Western Satrapies 546-330 BC. Procedings of an International Symposium at the Swedish Research Institute in Istanbul, 7-8 September 2017 », Abstracta Iranica [En ligne], Volume 42-43 | 2021, document 68, mis en ligne le 30 décembre 2021, consulté le 13 décembre 2022. URL : http://journals.openedition.org/ abstractairanica/53551 ; DOI : https://doi.org/10.4000/abstractairanica.53551

Ce document a été généré automatiquement le 13 décembre 2022.

Tous droits réservés 


\section{Ashk P. Dahlén. Achaemenid Anatolia: Persian Presence and Impact in the Western Satrapies 546-330 BC. Procedings of an International Symposium at the Swedish Research Institute in Istanbul, 7-8 September 2017}

Rémy Boucharlat

\section{RÉFÉRENCE}

Ashk P. Dahlén. Achaemenid Anatolia: Persian Presence and Impact in the Western Satrapies 546-330 BC. Procedings of an International Symposium at the Swedish Research Institute in Istanbul, 7-8 September 2017. 2020, (Boreas, Uppsala Studies in Ancient Mediterranean and Near Eastern Civilizations 37), Uppsala Universitet, 267 p. ill. (en libre accès).

1 Félicitons d'abord la maison d'édition et l'éditeur scientifique d'avoir mis très rapidement ce volume à disposition de tous les lecteurs intéressés.

2 Le titre est explicite, puisque dix des treize contributions concernent directement l'Anatolie. Le sous-titre est important, indiquant une thématique : "presence" est un terme assez neutre qui dérive d'observations, de même que "intercultural contacts in the Achaemenid Empire". En revanche "impact" est actif ; il a souvent pour équivalent "influence". Ces termes sont très largement employés dans l'historiographie de l'empire achéménide, avec une signification variable selon les régions, mais particulièrement pour l'Asie Mineure, territoire dans lequel les recherches de terrain, 
les synthèses et les colloques ont été particulièrement nombreux par rapport à d'autres régions de l'empire (voir la liste impressionnante dans la note 10, p. 10 de l'article introductif de P. Briant, bien nommé "On 'Achaemenid Impact' in Anatolia (reading notes)". "Impact" versus "dearth of archaeological documentation" ou "elusive empire" pour certains historiens sont des termes qui courent explicitement ou non à travers les contributions à ce volume.

3 Les «notes de lecture " de P. Briant reprennent en anglais, mais dans une version étendue et révisée, un texte publié en français en 2015. Elles tiennent lieu de solide introduction à la question de l'impact achéménide en Anatolie mais les "case studies" débordent la région, touchant le Caucase et incidemment l'Égypte et l'Arabie Saoudite.

4 Parmi les contributions en archéologie et objets, E.R.M. Dusinberre est particulièrement bien armée pour traiter de manière synthétique "Impacts of Empire in Achaemenid Anatolia". S. Berndt s'attache à une pièce de couvre-chef (tiara, kurbasia, kidaris), que seul le roi perse porterait en position relevée, fhypothèse aisant appel à l'iconographie et aux textes.

5 J. Blid propose une nouvelle interprétation de l'acrotère en forme de sphinx de l'andron (salle de banquet) de Mausole à Labraunda. Le même site a livré le bas-relief d'un chariot que tirent deux chevaux. Plutôt que la base d'une statue de victoire, P. Hellström suggère que la statue (manquante) qui s'élevait sur le chariot aurait été une statue de Zeus Labraundos.

6 Ch. Gates offre un panorama actualisé de la Cilicie à l'époque achéménide, principalement sept sites, après les synthèses publiées dans la collection Persika, celle de O. Casabonne (Persika 3, 2004) et la sienne propre (Persika 6, 2005).

7 M. Seyer étudie l'ensemble des tombes-piliers si caractéristique de la Lycie qui apparaissent au moment de la conquête perse. La plus ancienne serait justement la tombe du dynaste sous la domination perse.

8 À partir des sources écrites principalement, A. Dahlén explore les passages d'Hérodote mentionnant les habitudes des Perses avec le vin, habitudes qu'expliquent, selon l'auteur, les différences de culture avec les Grecs, mais pour qui le vin est du luxe.

$9 \quad$ A. Hultgård s'attaque à l'image et à la fonction de la déesse Anāhitā et son évolution en Asie Mineure où elle garde des aspects de son origine iranienne, mais qui adopte un langage commun aux religions d'Asie Mineure.

10 J. Köster reprend le récit de la révolte ionienne chez l'inépuisable Hérodote s'intéressant ici aux attitudes et aux ambitions de certains chefs ou élites locales.

11 L.G. Mitchell nuance le sens des conquêtes de Cyrus en Asie Mineure : la soumission des cités et états est d'abord un désir d'obtenir des pays pacifiés, le roi n'hésitant pas à négocier avec les élites.

12 R. Stoneman compare l'histoire de Cyrus, dans l'épisode avec Crésus, chez Hérodote moins favorable à celui-ci que le récit de Xanthus de Lydie influencé par la tradition des contes originaire de perse

13 Contents

14 - Ashk P. Dahlén, Preface

15 - Pierre Briant, "On "Achaemenid impact" in Anatolia (reading notes)"

16 - Elspeth R.M. Dusinberre, "Impacts of empire in Achaemenid Anatolia" 
17 - Susanne Berndt, "The upright tiara of the Persian king"

18 - Jesper Blid, "The andron of Maussollos at Labraunda and its architectural sculpture"

19 - Ashk P. Dahlén, "Living the Iranian dolce vita: Herodotus on wine drinking and luxury among the Persians"

20 - Charles Gates, "Cilicia, 550-330 BC: Persians and locals"

21 - Pontus Hellström, "A chariot at Labraunda"

22 - Anders Hultgård, "Invoking Anāhitā - from Iran to Asia Minor"

23 - Jan Köster, “Failed ambitions: Herodotus' account of the Ionian revolt and its motivation"

24 - Lynette G. Mitchell, “ "What age were you when the Mede came?” Cyrus the Great and Western Anatolia"

25 - Martin Seyer, "Pillar tombs and the Achaemenid rule in Lycia"

26 - Richard Stoneman, "Xanthus of Lydia, Aesop and Persian storytelling"

27 - Elspeth R.M. Dusinberre, “Concluding remarks".

\section{AUTEURS}

\section{RÉMY BOUCHARLAT}

UMR 5133 CNRS-Université de Lyon 